\title{
BOUNDARY CONDITIONS IN THE INFINITE INTERVAL AND SOME RELATED RESULTS
}

\author{
BY \\ RAO V. GOVINDARAJU( $\left.{ }^{1}\right)$
}

\begin{abstract}
The number of square-integrable solutions of a real, selfadjoint differential equation are determined using exclusively the elementary theory of matrices. Boundary conditions in the infinite interval are given a simple format and a relation between any two selfadjoint boundary conditions is deduced. Finally a lemma due to Titchmarsh, which forms the basis of eigenfunction expansions, is generalized.
\end{abstract}

We consider the case when the differential operator $L$ is real and formally selfadjoint. The order $n$ of $L$ is necessarily even. Let $n=2 v$. Very little is known about the algebra of boundary conditions in the interval $[0, \infty)$ except that they may be expressed by means of functions of $D^{*}$ and that the number of selfadjoint boundary conditions depends on the number of square-integrable solutions. In this paper the following results are proved:

(1) When the linearly independent solutions, square-integrable in $[0, \infty)$, of $L y+\mu y=0$ are $\nu+r, 0 \leqq r \leqq \nu$. Selfadjoint boundary conditions in $[0, \infty)$ can be expressed by means of a $(n+2 r) \times(\nu+r)$ matrix of rank $v+r$ which satisfies the condition

$$
M^{*}{ }_{v} J_{r} M=0_{v+r} \quad \text { where }{ }_{v} J_{r}=\left[\begin{array}{cc}
J_{v} & 0 \\
0 & J_{r}
\end{array}\right] \text { and } J_{s}=\left[\begin{array}{cc}
0 & -I_{s} \\
I_{s} & 0
\end{array}\right] .
$$

Further, the number of conditions involving zero alone (initial conditions) and the number of conditions involving infinity alone (final conditions) can be determined by knowing the structure of $M$.

(2) The difference between the number of initial conditions and the number of final conditions in a set of selfadjoint boundary conditions is a constant, equal to $\nu-r$.

(3) If $M_{1}$ and $M_{2}$ define two sets of real selfadjoint boundary conditions in $[0, \infty)$, then there exists a ${ }_{v} J_{r}$-symplectic matrix $S$ such that $M_{1}=S M_{2}$.

Received by the editors September 20, 1968 and, in revised form, May 15, 1970.

AMS 1969 subject classifications. Primary 3430.

Key words and phrases. Selfadjoint differential operators, selfadjoint boundary conditions, fundamental set of solutions, eigenvalues, symplectic matrices, Everitt surfaces, circles in matrix space.

(1) Supported in part by the Science Development Grant of the National Science Foundation at the University of Maryland. Sponsored by the University Grants Commission, India. Research done under the guidance of Professor S. Minakshisundaram at Andhra University, Waltair, India. 
(1) shows that we need no longer distinguish between boundary conditions in the infinite interval and boundary conditions in the finite interval. This result can be used to prove eigenfunction expansions using calculus of residues. W. N. Everitt [1] constructed the domains of the associated selfadjoint operator in the two cases when $r=0$ and $r=\nu$. In the rest of the cases Lemma 2.3 of [2] remained difficult to prove. The equivalent of this lemma is proved in this paper. The proof becomes simplified once we observe that we are no longer distinguishing between the point at infinity and a finite point as far as boundary conditions are concerned. We also give a method by which, given a certain type of selfadjoint real boundary conditions, we can find the corresponding point on the boundary of the surface $S_{r}(\infty, \mu)$ of Everitt [1].

(2) is a result known to be true in the finite interval. The equivalent of (3) for the finite interval is also true and the proof follows on similar lines.

1. Notations. $\int_{0}^{b} f \bar{g} d x$ is denoted by $(f, g)_{b}$ and $(f, g)$ denotes $(f, g)_{\infty} .\langle f, g\rangle_{b}$ denotes $(L f, g)_{b}-(f, L g)_{b} .\langle f, g\rangle$ denotes $\langle f, g\rangle_{\infty}$.

If $U(x)=\left[u_{1}(x) u_{2}(x) \cdots u_{s}(x)\right]$ and $V(x)=\left[v_{1}(x) v_{2}(x) \cdots v_{r}(x)\right]$ then the matrix $\left[\left\langle u_{i} v_{j}\right\rangle_{b}\right]_{1 \leqq i \leqq s ; 1 \leqq j \leqq r}$ is denoted by $\langle U, V\rangle_{b}$.

$\phi^{i}(x)$ denotes the $i$ th derivative of $\phi(x)$ and the matrix $\left[\phi_{j}^{i-1}(x)\right]_{1 \leqq i \leqq n ; 1 \leqq j \leqq m}$ is denoted by $W\left[\phi_{1} \phi_{2} \cdots \phi_{m}\right](x)$. The matrix $\left[\int \phi_{i j}(x) d x\right]$ is denoted by $\int\left[\phi_{i j}(x)\right] d x$. The identity matrix of order $r$ is denoted by $I_{r}$ and the $r \times s$ zero matrix by $0_{r s}$. $A^{\prime}$ denotes the transpose and $A^{*}$ denotes the conjugate transpose of $A$. $A<0$ means that $A$ is negative definite.

The differential operator $L$ is given by

$$
L y(x)=\frac{d^{v}}{d x^{v}}\left[q_{0}(x) y^{v}(x)\right]+\frac{d^{v-1}}{d x^{\nu-1}}\left[q_{1}(x) y^{v-1}(x)\right]+\cdots+q_{v}(x) y(x),
$$

$q_{0}(x), q_{1}(x)_{1}, \ldots, q_{v}(x)$ are real and $q_{0}(x) \neq 0$ for any $x . C^{n}[I]$ denotes the set of all complex valued functions having $n$ continuous derivatives on $I$.

$q_{i}(x)$ is in $C^{v-i}[0, b]$ for every finite $b>0$.

If $f(x)$ and $g(x)$ are in $C^{r}[0, b]$ then $\langle f, g\rangle_{b}=B[f, g](b)-B[f, g](0)$ where $B[f, g](x)$ is the bilinear form $W^{*}[g(x)] B(x) W[g(x)]$ where $B(x)$ is a real, skewsymmetric, nonsingular matrix associated with the operator $L[3$, p. 285]. Also $i B(x)$ has signature zero. (See Lemma 2 of [6].)

2. The fundamental set of solutions. $\mu$ is a complex number, $\operatorname{Im} \mu=v>0$.

THEOREM 2.1. We can choose a fundamental set of solutions of

$$
L y+\mu y=0,
$$

say $\theta(x, \mu)=\left[\theta_{1}(x, \mu) \theta_{2}(x, \mu) \cdots \theta_{v}(x, \mu)\right]$ and $\phi(x, \mu)=\left[\phi_{1}(x, \mu) \phi_{2}(x, \mu) \cdots \phi_{v}(x, \mu)\right]$ such that if $W[\theta(x, \mu)]=F(x, \mu)$ and $W[\phi(x, \mu)]=H(x, \mu)$ then

$$
[F H]^{\prime}(x, \mu) B(x)[F H](x, \mu)=J_{v}
$$

for any $x, 0 \leqq x<\infty$. 
Proof. Since $i B(x)$ has signature zero, there exists a real matrix $A$ such that $A^{\prime} B(0) A=J_{v}$. We can now choose a fundamental set of solutions $\theta(x, \mu), \phi(x, \mu)$ such that $[F H](0, \mu)=A$ so that (2.2) is satisfied at zero. But since $\langle\theta, \phi\rangle_{x}=0,(2.2)$ follows.

THEOREM 2.2. For any finite $x, i\left(H^{*} B H\right)(x, \mu)$ and $i\left(F^{*} B F\right)(x, \mu)$ are positive definite and

$$
\begin{aligned}
{\left[\left(F^{*} B F\right)+\left(H^{\prime} B \bar{H}\right)^{-1}\right](x, \mu) } & =\left[\left(F^{*} B H\right)\left(H^{*} B H\right)^{-1}\left(H^{*} B F\right)\right](x, \mu), \\
{\left[\left(H^{*} B F\right)\left(H^{\prime} B \bar{H}\right)\right](x, \mu) } & =\left[\left(H^{*} B H\right)\left(F^{\prime} B \bar{H}\right)\right](x, \mu) .
\end{aligned}
$$

Proof. Since $\langle(\theta, \phi),(\theta, \phi)\rangle_{x}=\left[(F H)^{*} B(F H)\right]_{0}^{x}(\mu)$ and since $(F H)(0, \mu)=A$ is real, we have $i\left(F^{*} B F\right)(x, \mu)=2 v \int_{0}^{x} \theta^{*} \theta d x$ and $i\left(H^{*} B H\right)(x, \mu)=2 v \int_{0}^{x} \phi^{*} \phi d x$ and as such, since $v>0$, are positive definite. Also

$$
\begin{aligned}
{\left[(F H)^{*} B(F H)\right](x, \mu) } & =\left[(F H)^{*} B(\bar{F} \bar{H})(\bar{F} \bar{H})^{-1} B^{-1}(F H)^{\prime} B(F H)\right](x, \mu) \\
& =J_{v}\left[(F H)^{\prime} B(\bar{F} \bar{H})\right]^{-1} J_{v} \quad \text { by }(2.2)
\end{aligned}
$$

calculating $\left[(F H)^{\prime} B(\bar{F} \bar{H})\right]^{-1}$ and comparing with the left side we get (2.3) and (2.4).

Let

$$
R_{b}(\mu)=-i\left(H^{*} B H\right)^{-1}(b, \mu)=\frac{1}{2 v}\left[\int_{0}^{b} \phi^{*} \phi d x\right]^{-1} .
$$

Then $R_{b}(\mu)>0$ and if $0<k_{1}(b, \mu) \leqq k_{2}(b, \mu) \leqq \cdots \leqq k_{v}(b, \mu)$ are the characteristic roots of $\int_{0}^{b} \phi^{*} \phi d x$ then there exists a unitary matrix $U_{b}(\mu)$ such that

$$
\left[U_{b}^{*} R_{b} U_{b}\right](\mu)=\frac{1}{2 v} \operatorname{Diag}\left[\frac{1}{k_{1}(b, \mu)}, \frac{1}{k_{2}(b, \mu)}, \cdots, \frac{1}{k_{v}(b, \mu)}\right],
$$

i.e. $R_{b}(\mu)=(1 / 2 v) P_{b}(\mu) P_{b}^{*}(\mu)$ where

$$
P_{b}(\mu)=U_{b}(\mu) \operatorname{Diag}\left[\frac{1}{\sqrt{ }\left(k_{1}(b, \mu)\right)}, \frac{1}{\sqrt{ }\left(k_{2}(b, \mu)\right)}, \cdots, \frac{1}{\sqrt{ }\left(k_{v}(b, \mu)\right)}\right] .
$$

Let $b$ tend to infinity through a sequence $b_{s}$. Then the sequences $k_{r}\left(b_{s}, \mu\right)$ are monotonic increasing [4] and hence converge either to a finite limit or diverge to infinity.

Let $k_{1}(b), k_{2}(b), \ldots, k_{r}(b)$ converge to finite limits $k_{1}(\mu), k_{2}(\mu), \ldots, k_{r}(\mu)$ as $b \rightarrow \infty$ through $b_{s}$. There exists a unitary matrix $U$ such that $\mathrm{Lt}_{s \rightarrow \infty} U_{b_{s}}=U$. (We apply Bolzano-Weierstrass theorem repeatedly to the bounded elements of the unitary matrix $U_{b}$.) We then have

$$
\underset{s \rightarrow \infty}{\operatorname{Lt}} R_{b_{s}}(\mu)=\underset{s \rightarrow \infty}{\operatorname{Lt}} \frac{1}{2 v} P_{b_{s}}(\mu) P_{b_{s}}^{*}(\mu)=\frac{1}{2 v} P(\mu) P^{*}(\mu)=R(\mu)
$$

where

$$
P(\mu)=U \cdot \operatorname{Diag}\left[\frac{1}{\sqrt{ }\left(k_{1}(\mu)\right)}, \frac{1}{\sqrt{ }\left(k_{2}(\mu)\right)}, \ldots, \frac{1}{\sqrt{ }\left(k_{r}(\mu)\right)}, 0, \ldots, 0\right]
$$


THEOREM 2.3. The $L_{2}[0, \infty)$-span of $\phi(x, \mu)$ is equal to the rank of $R(\mu)$.

Proof. The rank of $R(\mu)$ is, by (2.5), the number of characteristic roots of $\int_{0}^{b} \phi^{*} \phi d x$ which converge to finite limits. Theorem 5 in [5] completes the proof.

By (2.5), only $r$ columns of $P(\mu)$ are nonzero and linearly independent. Denoting these by $\mathscr{P}(\mu)$ we have $P(\mu)=[\mathscr{P}(\mu) 0]$.

THEOREM 2.4. The elements of $\phi(x, \mu) \mathscr{P}(\mu)$ are square-integrable.

Proof. Denoting the columns of $U$ by $\left[u_{1} u_{2} \cdots u_{r}\right]$, we have

$$
\underset{s \rightarrow \infty}{\operatorname{Lt}} \int_{0}^{b_{s}}\left[u_{1} \cdots u_{r}\right]^{*} \phi^{*} \phi\left[u_{1} \cdots u_{r}\right]=\operatorname{Diag}\left(k_{1}, k_{2}, \ldots, k_{r}\right)
$$

so that the elements of $\phi(x, \mu) \mathscr{P}(\mu)$ will be in $L_{2}[0, \infty)$. Further

$$
\int_{0}^{\infty} \mathscr{P} \phi^{*} \phi \mathscr{P} d x=I_{r}
$$

and since

$$
-2 i \int_{0}^{b} \phi^{*}(x, i) \phi(x, i) d x=\left[H^{*} B H\right]_{0}^{b}(i)=\left(H^{*} B H\right)(b, i),
$$

we have

$$
\left[\mathscr{P} * H^{*} B H \mathscr{P}\right](\infty, i)=-2 i I_{r}
$$

or

$$
\left[\overline{\mathscr{P}} \mathscr{P}^{*} H^{*} B H \mathscr{P}^{\prime}\right](\infty, i)=-2 i \overline{\mathscr{P}}(i) \mathscr{P}^{\prime}(i)
$$

3. Circles in the matrix space $\left({ }^{2}\right)$. Let $\chi$ be any $n \times \nu$ real matrix of rank $\nu$ such that $\chi^{\prime} B(b) \chi=0_{v}$. Then any function $y(x) \in C^{n-1}[0, b]$ satisfying the condition

$$
\chi^{\prime} B(b) W[y(b)]=0_{v, 1}
$$

has the property $W^{\prime}[y(b)] B(b) W[y(b)]=0$.

We shall find a $\nu$ th ordered matrix $Z_{b}(\mu)\left({ }^{3}\right)$ such that the elements of the vector $\psi_{b}(x, \mu)=\theta(x, \mu)+\phi(x, \mu) Z_{b}(\mu)$ satisfy $(3.1)$, i.e. if $G_{b}(x, \mu)=W\left[\psi_{b}(x, \mu)\right]=F(x, \mu)$ $+H(x, \mu) Z_{b}(\mu)$, then

$$
\chi^{\prime} B(b) G_{b}(x, \mu)=0_{v}
$$

( $\left.{ }^{2}\right)$ Work on these lines was previously done by Kodaira [7] using geometry, by Everitt [8] discussing the problem when $n=4$ and generalizing later in [9].

$\left(^{3}\right)$ It may be noted that the $r$ th column of $Z_{b}(\mu)$ is essentially an element on the surface $S_{r}(b, \mu)$ of Everitt [1]. 
THEOREM 3.1. The eigenvalues of the boundary value problem

$$
L y+\mu y=0, \quad W^{\prime}[y(0)] B(0) H(0)=0, \quad W^{\prime}[y(b)] B(b) \chi=0
$$

are real and are the roots of $\operatorname{det}\left[H^{\prime}(b, \mu) B(b) \chi\right]=\Delta(\mu)=0$ and conversely.

Proof. Let $y(x, s)=\theta(x, s) \beta_{1}+\phi(x, s) \beta_{2}$ be an eigenfunction. Applying the condition at zero, we get $\beta_{1}=0$. The condition at $b$ gives $\left[\chi^{\prime} B(b) H^{\prime}(b, s)\right] \beta_{1}=0$ so that in order that $\beta_{2} \neq 0, \Delta(s)=0$. Now consider

$$
[H(b, \mu) \chi]^{\prime} B(b)[H(b, \mu) \chi]=\left[\begin{array}{cc}
0 & H^{\prime}(b, \mu) B(b) \chi \\
\chi^{\prime} B(b) H(b, \mu) & 0
\end{array}\right] .
$$

$\Delta(s)=0$ implies $[H(b, s) \chi]$ is singular so that there exist nonzero $\nu \times 1$ vectors $\alpha$ and $\beta$ such that $\chi \alpha=H(b, s) \beta$. This shows that the solution $\phi(x, s) \beta$ satisfies the boundary conditions so that $s$ is an eigenvalue. That the eigenvalues are real is well known. Hence for nonreal $\mu$, the matrix $\chi^{\prime} B(b) H(b, \mu)$ is nonsingular so that by (3.2), with $\operatorname{Im} \mu=v>0$,

$$
Z_{b}(\mu)=-\left[\chi^{\prime} B(b) F(b, \mu)\right]\left[\chi^{\prime} B(b) H(b, \mu)\right]^{-1} .
$$

Since $\chi$ is independent of $\mu$ we also have $\chi^{\prime} B(b) G_{b}\left(x, \mu^{\prime}\right)=0_{\nu}$. Taking $\mu^{\prime}=\mu$ we have $Z_{b}^{\prime}(\mu)=Z_{b}(\mu)$ and for $\mu^{\prime}=\bar{\mu}$,

$$
G_{b}^{*}(b, \mu) B(b) G_{b}(b, \mu)=0_{v} .
$$

For any $Z_{b}(\mu)$ satisfying (3.4)

$$
2 i v \int_{0}^{b} \psi_{b}^{*} \psi_{b} d x=\bar{Z}_{b}(\mu)-Z_{b}(\mu)
$$

Hence, $\operatorname{Im} Z_{b}(\mu)<0$ so that $Z_{b}(\mu)$ is nonsingular. (3.4) can be written, using (2.3) and (2.4), as

$$
\left[Z_{b}(\mu)-\mathscr{C}_{b}(\mu)\right]^{*} R_{b}^{-1}(\mu)\left[Z_{b}(\mu)-\mathscr{C}_{b}(\mu)\right]-R_{b}^{\prime}(\mu)=0
$$

where $\mathscr{C}_{b}(\mu)=-\left[\left(H^{*} B H\right)^{-1}\left(H^{*} B F\right)\right](b, \mu)$ and $\mathscr{C}_{b}^{\prime}(\mu)=\mathscr{C}_{b}(\mu)$ by (2.4). Further

$$
-2 \cdot \operatorname{Im} \mathscr{C}_{b}(\mu)=2 v \int_{0}^{b}\left(\theta+\phi \mathscr{C}_{b}\right) *\left(\theta+\phi \mathscr{C}_{b}\right) d x+R_{b}^{\prime}(\mu)
$$

The right side of (3.7) is positive definite so that $\operatorname{Im} \mathscr{C}_{b}(\mu)<0$ which implies that $\mathscr{C}_{b}(\mu)$ is nonsingular.

As we take different conditions at $b$ by changing $\chi$ we get a system of $Z_{b}(\mu)$ satisfying (3.5) and (3.6). (3.6) is the equation of a "circle" $C_{b}(\mu)$ with "radius" $R_{b}(\mu)$ and center $\mathscr{C}_{b}(\mu)$ in the space of symmetric matrices of order $\nu$.

Definition 3.1. Let $C$ be any circle in a space of matrices given by the equation $f(Z)=0$. We say that a matrix $A$ lies inside $C$ if $A$ is in the space and $f(A)<0$.

THEOREM 3.2. The circle $C_{b}(\mu)$ decreases as $b$ increases, i.e., if $a>b$ then every matrix on $C_{a}(\mu)$ lies inside $C_{b}(\mu)$. 
Proof. If $Z_{a}(\mu)$ lies on $C_{a}(\mu)$, then by (3.5)

$$
-v \int_{0}^{a} \psi_{a}^{*} \psi_{a} d x=\operatorname{Im} Z_{a}(\mu)
$$

Also since

$$
\langle(\theta, \phi),(\theta, \phi)\rangle_{b}=[F H]^{*}(b, \mu) B(b)[F H](b, \mu)-J_{v},
$$

we have, premultiplying by $\left[I Z_{a}^{*}\right]$ and postmultiplying by $\left[Z_{a}^{I}\right]$,

$$
-2 i v \int_{0}^{b} \psi_{a}^{*} \psi_{a} d x-2 i \operatorname{Im} Z_{a}(\mu)=\left[\left(F+H Z_{a}\right)^{*} B\left(F+H Z_{a}\right)\right](b, \mu) .
$$

The right side is $-i\left[\left(Z_{a}-\mathscr{C}_{b}\right)^{*} R_{b}^{-1}\left(Z_{c}-\mathscr{C}_{b}\right)\right]+i R_{b}^{\prime}(\mu)$ while the left side equals, by (3.8), 2iv $\int_{b}^{a} \psi_{a}^{*} \psi_{a} d x$ from which the theorem follows.

THEOREM 3.3. A matrix $Z$ lies on the circle $C_{b}(\mu)$ if and only if it can be written as

$$
Z(\mu)=(1 / 2 v) P_{b}(\mu) \mathscr{U} P_{b}^{\prime}(\mu)+\mathscr{C}_{b}(\mu)
$$

where $\mathscr{U}$ is a symmetric unitary matrix.

Proof. Since $R_{b}(\mu)=(1 / 2 v) P_{b}(\mu) P_{b}^{*}(\mu)$, the circle $C_{b}(\mu)$ can be written as

$$
4 v^{2}\left(Z_{b}-\mathscr{C}_{b}\right){ }^{*} P_{b}^{*-1} P_{b}^{-1}\left(Z_{b}-\mathscr{C}_{b}\right)-\bar{P}_{b} P_{b}^{\prime}=0 .
$$

Let $Z$ be any matrix and let $2 v \cdot P_{b}^{-1}(\mu)\left[Z-\mathscr{C}_{b}\right] P_{b}^{\prime-1}(\mu)=\mathscr{U}$.

If $Z$ lies on $C_{b}(\mu)$, it will be symmetric and satisfy (3.9) so that $\mathscr{U}$ will be symmetric and unitary. The converse is also true. Hence, the theorem.

THEOREM 3.4. The elements of any matrix $Z$ lying inside $C_{b}(\mu)$ are bounded.

Proof. As in Theorem 3.3, we can show that any matrix $Z$ lying inside $C_{b}(\mu)$ can be written as

$$
Z=(1 / 2 v) P_{b}(\mu) V P_{b}^{\prime}(\mu)+\mathscr{C}_{b}(\mu)
$$

where $V$ is a symmetric matrix and $V^{*} V<I_{\nu}$ (i.e., $V^{*} V-I_{v}<0$ ).

For any $b>k$, the circle $C_{b}(\mu)$ lies inside $C_{k}(\mu)$ and so will be the centre $\mathscr{C}_{b}(\mu)$ of $C_{b}(\mu)$. Hence, by Theorem 3.4 the elements of $\mathscr{C}_{b}(\mu)$ will be bounded, the bounds being independent of $b$. A repeated application of Bolzano-Weierstrass theorem shows that there exists a matrix $\mathscr{C}(\mu)$ to which $\mathscr{C}_{b}(\mu)$ converges through a diverging sequence. Without loss of generality we can assume that this sequence is the same as the sequence $b_{s}$ through which $P_{b}(\mu)$ tends to $P(\mu)$.

Let $Z_{b}(\mu)$ be any matrix on the circle $C_{b}(\mu)$ given by

$$
Z_{b}(\mu)=(1 / 2 v) P_{b}(\mu) \mathscr{U} P_{b}^{\prime}(\mu)+\mathscr{C}_{b}(\mu) .
$$

Letting $b$ tend to infinity through $b_{s}$, the matrix $Z_{b}(\mu)$ tends to a matrix $Z(\mu)$ given by $Z(\mu)=(1 / 2 v) P(\mu) \mathscr{U} P^{\prime}(\mu)+\mathscr{C}(\mu)$. Note that the symmetric unitary matrix $\mathscr{U}$ is 
just a parameter and so does not change in the limit. Also by (3.8),

$$
\int_{0}^{\infty} \psi^{*} \psi d x=-\frac{1}{v} \operatorname{Im} Z(\mu)
$$

where $\psi(x, \mu)=\theta(x, \mu)+\phi(x, \mu) Z(\mu)$.

Each matrix $Z_{b}$ on $C_{b}(\mu)$ tends to a matrix $Z(\mu)$ which lies on a circle $C(\mu)$ called the limiting circle with centre $\mathscr{C}(\mu)$ and radius $R(\mu)=P(\mu) P^{*}(\mu)$. When the rank of $R(\mu)$ is $r$ we call $C(\mu)$ as the limit circle of type $r$. When $r=0$, the circle reduces to a single matrix $\mathscr{C}(\mu)$ and the case is called limit point case.

It will be shown in the next section that $r$ is directly connected with the number of solutions in $L_{2}[0, \infty)$ of $L y+\mu y=0$ and it is known that this number is independent of $\mu$ when it lies in one of the half-planes: $\operatorname{Im} \mu>0$ or $\operatorname{Im} \mu<0$. But since $L$ is real, $\bar{y}(x, \mu)=y(x, \bar{\mu})$ so that the number of solutions in $L_{2}[0, \infty)$ is independent of $\mu$ and hence so is the type of the limiting circle.

\section{Square-integrable solutions.}

THEOREM 4.1. There exist at least $\nu$ linearly independent solutions of $L y+\mu y=0$, $\operatorname{Im} \mu=v>0$ in $L_{2}[0, \infty)$.

Proof. By letting $b$ tend to infinity through $b_{s}$ in (3.7), we have

$$
2 v \int_{0}^{\infty}(\theta+\phi \mathscr{C})^{*}(\theta+\phi \mathscr{C}) d x=-2 \operatorname{Im} \mathscr{C}(\mu)-\frac{1}{2 v} \bar{P}(\mu) P^{\prime}(\mu) .
$$

This shows that the elements of $(\theta+\phi \mathscr{C})(x, \mu)$ are in $L_{2}[0, \infty)$.

THEOREM 4.2. In case of limit circle of type $r$, there exist exactly $\nu+r$ solutions of $L y+\mu y=0, \operatorname{Im} \mu>0$ in $L_{2}[0, \infty)$.

Proof. When the rank of $R(\mu)$ is $r$, the $L_{2}[0, \infty)$-span of $\phi(x, \mu)$ is, by Theorem 2.3, $r$ and the elements of $\phi(x, \mu) \mathscr{P}(\mu)$ are in $L_{2}[0, \infty)$. By Theorem 4.1, the elements of $\theta(x, \mu)+\phi(x, \mu) \mathscr{C}(\mu)$ are in $L_{2}[0, \infty)$. It only remains to show that these two sets of solutions are linearly independent and this follows from the fact that the matrix

is of rank $\nu+r$.

$$
\left[\begin{array}{cc}
I_{\nu} & 0_{v r} \\
\mathscr{C}(\mu) & \mathscr{P}(\mu)
\end{array}\right]
$$

Let $\pi(x, \mu)=\theta(x, \mu) \alpha+\phi(x, \mu) \beta$ be any solution in $L_{2}[0, \infty)$ where $\alpha$ and $\beta$ are $\nu+1$ vectors. If $\alpha=0$ by Theorem 2.3, $\pi(x, \mu)=\phi(x, \mu) \beta$ will be in the space spanned by $\phi(x, \mu) \mathscr{P}(\mu)$. If $\alpha \neq 0$, then $\theta(x, \mu) \alpha+\phi(x, \mu) \mathscr{C}(\mu) \alpha$ will be a nontrivial solution in $L_{2}[0, \infty)$ so that $\phi(x, \mu)[\beta-\mathscr{C}(\mu) \alpha]$ will be in $L_{2}[0, \infty)$ so that by Theorem 2.3 there is a $\nu \times 1$ vector $\sigma$ such that $\beta-\mathscr{C}(\mu) \alpha=\mathscr{P}(\mu) \sigma$. Since

$$
\left[\begin{array}{cc}
I_{v} & 0_{v r} \\
\mathscr{C}(\mu) & \mathscr{P}(\mu)
\end{array}\right]\left[\begin{array}{l}
\alpha \\
\sigma
\end{array}\right]=\left[\begin{array}{l}
\alpha \\
\beta
\end{array}\right]
$$

which shows that $\pi(x, \mu)$ is a linear combination of $\phi(x, \mu) \mathscr{P}(\mu)$ and $\theta(x, \mu)$ $+\phi(x, \mu) \mathscr{C}(\mu)$. 
5. A basis of $D^{*} \bmod D_{0} . \quad D^{*}$ is the set of all functions $f(x)$ such that $f(x)$ is continuously derivable $n-1$ times and $f^{n-1}(x)$ is absolutely continuous and $f(x)$ and $L f(x)$ are in $L_{2}[0, \infty)$.

$D_{0}$ is the set of all functions of $g(x)$ in $D^{*}$ such that $\langle f, g\rangle_{\infty}=0$ for every $f(x)$ in $D^{*}$.

$D^{+}$denotes the set of all solutions of $L y+i y=0$ in $L_{2}[0, \infty)$ and $D^{-}$denotes the set of all solutions of $L y-i y=0$ in $L_{2}[0, \infty)$. That $D^{*}=D_{0}+D^{+}+D^{-}$is well known.

Throughout we assume that the limiting circle is of type $r$. Any matrix $Z(i)$ on the limiting circle $C(i)$ is given by

$$
Z(i)=\frac{1}{2} P(i) \mathscr{U} P^{\prime}(i)+\mathscr{C}(i)
$$

and is such that

$$
\left[(F+H Z)^{*} B(F+H Z)\right](\infty, i)=0 .
$$

Taking $i I,-i I$ and $I$ in place of $\mathscr{U}$ in (5.1), we get the following equations from (5.2):

$$
\begin{aligned}
{\left[(F+H \mathscr{C})^{*} B(F+H \mathscr{C})-\right.} & (i / 2) \bar{P} P^{*} H^{*} B(F+H \mathscr{C}) \\
& \left.+(i / 2)(F+H \mathscr{C})^{*} B H P P^{\prime}+\frac{1}{4} \bar{P} P^{*} H^{*} B H P P^{\prime}\right](\infty, i)=0 \\
{\left[(F+H \mathscr{C})^{*} B(F+H \mathscr{C})+\right.} & (i / 2) \bar{P} P^{*} H^{*} B(F+H \mathscr{C}) \\
& \left.-(i / 2)(F+H \mathscr{C})^{*} B H P P^{\prime}+\frac{1}{4} \bar{P} P^{*} H^{*} B H P P^{\prime}\right](\infty, i)=0 \\
{\left[(F+H \mathscr{C})^{*} B(F+H \mathscr{C})+\right.} & \frac{1}{2} \bar{P} P^{*} H^{*} B(F+H \mathscr{C}) \\
& \left.+\frac{1}{2}(F+H \mathscr{C})^{*} B H P P^{\prime}+\frac{1}{4} \bar{P} P^{*} H^{*} B H P P^{\prime}\right](\infty, i)=0
\end{aligned}
$$

Note that each term in the brackets in each of these equations is finite. Hence, from (5.3) and (5.4) we get by adding, by (2.8),

(5.6) $\left[(F+H \mathscr{C})^{*} B(F+H \mathscr{C})\right](\infty, i)=-\frac{1}{4}\left[\bar{P} P^{*} H^{*} B H P P^{\prime}\right](\infty, i)=(i / 2) \overline{\mathscr{P}} \mathscr{P}{ }^{\prime}(i)$

and by (5.4) and (5.5) we have

$$
\left[\bar{P} P^{*} H^{*} B(F+H \mathscr{C})\right](\infty, i)=0,
$$

i.e.

i.e. by (2.5)

$$
P\left[\begin{array}{c}
\mathscr{P}^{*} H^{*} B(F+H \mathscr{C}) \\
0_{v-r, v}
\end{array}\right]=0_{v}
$$

$$
\operatorname{Diag}\left(\frac{1}{\left(k_{1}\right)^{1 / 2}}, \frac{1}{\left(k_{2}\right)^{1 / 2}}, \ldots, \frac{1}{\left(k_{r}\right)^{1 / 2}}, 0, \ldots, 0\right)\left[\begin{array}{c}
\mathscr{P} * H^{*} B(F+H \mathscr{C}) \\
0
\end{array}\right]=0_{v},
$$

i.e.

$$
\left[\mathscr{P}^{*} H^{*} B(F+H \mathscr{C})\right]=0_{r, v}
$$


THEOREM 5.1. The matrix $Q(i)=\operatorname{Im} \mathscr{C}(i)+\mathrm{Rl} R(i)$ is nonsingular.

Proof. Let

$$
s(x)=[\phi(x, i) \mathscr{P}(i) \delta(x, i) \overline{\mathscr{P}}(i)(\theta+\phi \mathscr{C})(x, i)(\bar{\theta}+\bar{\phi} \mathscr{C})(x, i)] .
$$

Then the elements of $s(x)$ form a basis of $D^{+}+D^{-}$(we say that $s(x)$ is a basis of $\left.D^{+}+D^{-}\right)$. We then have

$$
B[s, s](0)=\left[\begin{array}{cccc}
0_{r} & 0_{r} & \mathscr{P} *(i) & \mathscr{P}^{*}(i) \\
0_{r} & 0_{r} & \mathscr{P}^{\prime}(i) & \mathscr{P}^{\prime}(i) \\
-\mathscr{P}(i) & -\overline{\mathscr{P}}(i) & -2 i \operatorname{Im} \mathscr{C}(i) & 0_{v} \\
-\mathscr{P}(i) & -\overline{\mathscr{P}}(i) & 0_{v} & 2 i \operatorname{Im} \mathscr{C}(i)
\end{array}\right]
$$

and by using (2.7), (5.6) and (5.7),

$$
B[s, s](\infty)=\left[\begin{array}{cccc}
-2 i I_{r} & 0_{r} & 0_{r, v} & P^{*}(i) \\
0_{r} & 2 i I_{r} & \mathscr{P}^{\prime}(i) & 0_{r, v} \\
0_{v, r} & -\overline{\mathscr{P}}(i) & i R^{\prime}(i) & 0_{v} \\
-\mathscr{P}(i) & 0_{v, r} & 0_{v} & -i R(i)
\end{array}\right] .
$$

Hence,

$$
\langle s, s\rangle=\left[\begin{array}{cccc}
-2 i I_{r} & 0_{r} & -\mathscr{P}^{*}(i) & 0_{r, v} \\
0_{r} & 2 i I_{r} & 0_{r, v} & -\mathscr{P}^{\prime}(i) \\
\mathscr{P}(i) & 0_{r, v} & i R^{\prime}(i)+2 i \operatorname{Im} \mathscr{C}(i) & 0_{v} \\
0_{r, v} & \mathscr{P}(i) & 0_{v} & -(i / 2) R(i)-2 i \operatorname{Im} \mathscr{C}(i)
\end{array}\right] .
$$

We note that the matrix $\langle s, s\rangle$ is nonsingular. For, if we assume the contrary, there exists a nonzero $(n+2 r) \times 1$ vector $\alpha$ such that $\langle s, s\rangle \alpha=0$, i.e. $\langle s, s \alpha\rangle=0$ which shows that the function $s(x) \alpha$ in $D^{+}+D^{-}$is such that $\langle f, s \alpha\rangle=0$ for all $f(x)$ in $D^{*}$ and this means that $s(x) \alpha$ is in $D_{0}$ which is a contradiction. Hence the matrix on the right of (5.10) is nonsingular. Premultiplying the first "row" of $\langle s, s\rangle$ by $\mathscr{P}(i) / 2 i$ and adding to the third, the third "row" becomes $\left[0_{v r} 0_{v r} 2 i Q(i) 0_{v}\right]$ which shows that $Q(i)$ is nonsingular.

THEOREM 5.2. There exists a real basis $p(x)$ of $D^{+}+D^{-}$such that

$$
B[p, p] 0=\left[\begin{array}{ccc}
0_{v} & Q(i) & 0_{v, 2 r} \\
-Q(i) & 0_{v} & 0_{v, 2 r} \\
0_{2 r, v} & 0_{2 r, v} & 0_{2 r}
\end{array}\right]
$$

and

$$
B[p, p](\infty)=\left[\begin{array}{cc}
0_{n} & 0_{n .2 r} \\
0_{2 r, n} & J_{r}
\end{array}\right]
$$


Proof. The basis $p(x)$ is given by $p(x)=s(x) \cdot A(i)$ where

$$
\begin{aligned}
A(i)=\left[\begin{array}{cccc}
I_{r} & -i I_{r} & -\frac{1}{2} \mathscr{P}^{*} & (1 / 2 i) \mathscr{P}^{*} \\
I_{r} & i I_{r} & -\frac{1}{2} \mathscr{P}^{\prime} & -(1 / 2 i) \mathscr{P}^{\prime} \\
0_{v, r} & 0_{v, r} & i I_{v} & I_{r} \\
0_{v, r} & 0_{v, r} & -i I_{v} & I_{v}
\end{array}\right] \\
\\
\times\left[\begin{array}{cccc}
0_{r, v} & \operatorname{Im} \mathscr{P}^{\prime} & I_{r} & 0_{r} \\
0_{r, v} & -\mathrm{R} 1 \mathscr{P}^{\prime} & 0_{r} & I_{r} \\
I_{v} & -Q^{-1} \operatorname{Im} R(i) & Q^{-1} \operatorname{R1} \mathscr{P}(i) & Q^{-1} \operatorname{Im} \mathscr{P}(i) \\
0_{v} & -I_{v} & 0_{v, r} & 0_{v, r}
\end{array}\right] .
\end{aligned}
$$

It can be seen that $A$ is the product of two nonsingular matrices and is hence nonsingular. We can verify that $p(x)$ is real and satisfies (5.11) and (5.12) by using (5.8) and (5.9).

Now since the matrix

$$
i\left[\begin{array}{cc}
0 & -Q(i) \\
Q(i) & 0
\end{array}\right]
$$

is skew-symmetric, nonsingular and has signature zero, there exists a real matrix $K$ such that

$$
K^{\prime}\left[\begin{array}{cc}
0 & -Q(i) \\
Q(i) & 0
\end{array}\right] K=J_{v}
$$

If

$$
t(x)=p(x)\left[\begin{array}{cc}
K & 0_{v, 2 r} \\
0_{2 r, v} & I_{2 r}
\end{array}\right]
$$

then by (5.11) and (5.12),

$$
\begin{aligned}
& B[t, t](0)=\left[\begin{array}{cc}
-J_{v} & 0_{n, 2 r} \\
0_{2 r, n} & 0_{2 r}
\end{array}\right], \\
& B[t, t](\infty)=\left[\begin{array}{cc}
0_{n} & 0_{n, 2 r} \\
0_{2 r, n} & J_{r}
\end{array}\right],
\end{aligned}
$$

and hence,

$$
\langle t, t\rangle={ }_{v} J_{r} .
$$

\section{Algebra of boundary conditions.}

Definition 6.1. A homogeneous boundary condition in the interval $[0, \infty)$ is a condition on $y(x)$ in $D^{*}$ of the form $\langle u, y\rangle=0$ where $u(x)$ is a fixed function in $D^{*}$.

The boundary conditions

$$
\left\langle u_{i}, y\right\rangle=0, \quad i=1,2, \ldots, s,
$$

are said to be linearly independent if $\sum_{1}^{s} a_{i}\left\langle u_{i}, y\right\rangle=0$ for every $y(x)$ of $D^{*}$ implies $a_{i}=0, i=1,2, \ldots, s$. 
The following theorem follows at once:

THEOREM 6.1. The boundary conditions (6.1) are linearly independent if and only if $u_{1}(x), u_{2}(x), \ldots, u_{s}(x) \bmod D_{0}$ are linearly independent.

Since $t(x)$ is a basis of $D^{*} \bmod D_{0}$, there exists a unique $(n+2 r) \times s$ matrix $M$ (assuming that the limit circle is of type $r$ ) such that

$$
\left[u_{1}(x), u_{2}(x), \ldots, u_{s}(x)\right]=t(x) M \bmod D_{0} .
$$

With this notation, we can denote the conditions (6.1) by $M y[0, \infty)=0$. If $y(x)$ in $D^{*}$ satisfies these conditions, then $y(x)=t(x) \beta$ where $\beta$ is a $(n+2 r) \times 1$ vector satisfying, by $(5.15), M^{*}{ }_{v} J_{r} \beta=0$. The conditions are said to be selfadjoint if $M^{*}{ }_{v} J_{r} M=0$.

THEOREM 6.2. Let

$$
M=\left[\begin{array}{ccc}
M_{1} & 0 & M_{3} \\
0 & M_{2} & M_{4}
\end{array}\right]
$$

where $M_{1}$ is $n \times p, M_{2}$ is $2 r \times q, M_{3}$ is $n \times s$, and $M_{4}$ is $2 r \times s . M_{1}$ and $M_{2}$ have ranks $p$ and $q$ respectively, and let the matrix $\left[\begin{array}{l}M_{3} \\ M_{4}\end{array}\right]$ have rank $s$. Then the conditions $M y[0, \infty)=0$ imply $p$ conditions involving zero alone, $q$ conditions involving $\infty$ alone and $s$ conditions involving both zero and infinity.

Proof. If $y(x)=t(x) \beta \bmod D_{0}$ then the conditions $M y[0, \infty)$ are equivalent to the three sets of conditions

$$
\left[M_{1}^{*} 0\right]_{v} J_{r} \beta=0, \quad\left[0 M_{2}^{*}\right]_{v} J_{r} \beta=0, \quad\left[M_{1}^{*} M_{2}^{*}\right]_{v} J_{r} \beta=0 .
$$

The first condition may be written as

$$
\left[M_{1}^{*} 0\right]\left[\begin{array}{cc}
-J_{v} & 0 \\
0 & 0_{2 r}
\end{array}\right] \beta=0,
$$

i.e. by (5.13), $\left[M_{1}^{*} 0\right] B[t, t](0) \cdot \beta=0$. If $t_{1}(x)=t(x)\left[\begin{array}{c}M_{1} \\ 0\end{array}\right]$ then the first set of conditions are equivalent to $B\left[t_{1}(x), y\right](0)=0$ which are $p$ linearly independent conditions involving zero alone (initial conditions).

Using (5.14) we can show in a similar way that the second set of conditions involve infinity alone (final conditions) given by $B\left[t_{2}(x), y\right](\infty)=0, t_{2}(x)=t(x)\left[{ }_{M_{2}}^{0}\right]$.

If $t_{3}(x)=t(x)\left[M_{4} M_{4}\right]$, the third set of conditions becomes $\left\langle t_{3}, y\right\rangle=0$ which are $s$ conditions involving both zero and infinity.

It can be easily seen that a selfadjoint domain of $L$ can be defined by means of $\nu+r$ linearly independent, homogeneous, selfadjoint boundary conditions. From now on, whenever we say "selfadjoint boundary conditions", we mean $v+r$ linearly independent homogeneous boundary conditions which are selfadjoint.

THEOREM 6.3. In a set of selfadjoint boundary conditions, the difference between the initial and final conditions is a constant, equal to $\nu-r$. 
Proof. By a series of elementary column transformations, which leave the conditions $M y[0, \infty)=0$ unaltered, we can reduce the matrix $M$ to the form given in Theorem 6.2. We will show that $p-q=\nu-r$. We can write $M=\left[\begin{array}{l}V_{1} \\ V_{2}\end{array}\right]$ where $V_{1}$ is $n \times(\nu+r)$ and has rank $\nu+r-q$ and $V_{2}$ is $2 r \times(\nu+r)$ and has rank $\nu+r-p$. There exists an $n \times(\nu-r+q)$ matrix $X$ of rank $\nu-r+q$ such that $V_{1}^{*} X=0$. Also since $M$ has rank $\nu+r$, there exists an $(n+2 r) \times(2 r-q)$ matrix $\left[{ }_{Y_{2}}^{Y_{1}}\right], Y_{1}$ is $n \times(2 r-q)$ and $Y_{2}$ is $2 r \times(2 r-q)$, such that the matrix $\left[\begin{array}{lll}X & 2 & Y \\ 0 & 1 & Y\end{array}\right]$ has rank $\nu+r$ and

$$
\left[\begin{array}{ll}
V_{1}^{*} & V_{2}^{*}
\end{array}\right]\left[\begin{array}{cc}
X & Y_{1} \\
0 & Y_{2}
\end{array}\right]=0 .
$$

Note that the rank of $Y_{2}$ is $2 r-q$. For, if it is less than $2 r-q$, some columns of $Y_{2}$ can be made zero by column transformations so that by (6.3) there exist more than $\nu-r+q$ linearly independent column vectors $\beta$ such that $V_{1}^{*} \beta=0$ which contradicts our assumption that $V_{1}$ has rank $\nu+r-q$. Since $M^{*}{ }_{\nu} J_{r} M=0$, by (6.3) it follows that there is a $(\nu+r)$ th ordered nonsingular matrix $T$ such that

$$
{ }_{v} J_{r} M=\left[\begin{array}{cc}
X & Y_{1} \\
0 & Y_{2}
\end{array}\right] T, \text { i.e. } M=-{ }_{v} J_{r}\left[\begin{array}{cc}
X & Y_{1} \\
0 & Y_{2}
\end{array}\right] T
$$

so that $V_{2}=-J_{r}\left[\begin{array}{ll}0 & Y_{2}\end{array}\right] T$. Comparing the ranks we have $\nu+r-p=2 r-q$ or $p-q=\nu-r$.

This theorem shows that when all the solutions are in $L_{2}[0, \infty]$ the number of initial conditions is equal to the number of final conditions which is known to be true in the case of finite intervals.

THEOREM 6.4. The number of conditions which include infinity are at least $r$ and at most $2 r$.

Proof. That the upper limit is $2 r$ is evident since $V_{2}$ is a $2 r \times v+r$ matrix. Substituting the value of $M$ given in (6.4) in $M^{*}{ }_{v} J_{r} M=0$, we get $X^{*} J_{v} X=0$ which shows that the rank of $X$ is at most $\nu$, i.e. $\nu-r+q \leqq \nu$ or $q \leqq r$. Hence, by Theorem $6.3, \nu-r=p-q \geqq p-r$ or $p \leqq \nu$. Hence, $\nu+r-p \geqq r$, i.e. rank of $V_{2}$ is at least $r$.

This theorem shows that in the case of limit point, each selfadjoint domain can be defined by a set of $v$ initial conditions.

We say that the boundary conditions are real if the boundary condition functions $u_{i}(x)$ are real, i.e., since $t(x)$ is real, if the matrix $M$ is real. We shall now deduce a relation between any two sets of real selfadjoint boundary conditions $M_{1} y[0, \infty)=0$ and $M_{2} y[0, \infty)=0$.

Definition 6.2. Let $K$ be any matrix of order $p$. A matrix $S$ of order $p$ is said to be a $K$-symplectic matrix if $S^{*} K S=K$.

THEOREM 6.5. Let $M$ be any $2 p \times p$ real matrix of rank $p$ such that $M^{\prime} K M=0$ for some nonsingular, skew-symmetric matrix $K$ of order $2 p$. Let $X$ be any nonsingular matrix of order $p$ and let $Y$ be any skew-symmetric matrix of order $p$. Then there exists a $2 p \times p$ matrix $N$ such that $[M N]$ is nonsingular and $M^{\prime} K N=X$ and $N^{\prime} K N=Y$. 
Proof. We shall denote the linearly independent columns of $X$ by $x_{1}, x_{2}, \ldots, x_{p}$. Consider the linear equations

$$
M^{\prime} K \beta_{1}=x_{1}
$$

where $\beta_{1}$ is a $2 p \times 1$ vector.

Since the rank of $M^{\prime} K=$ rank of $\left[M^{\prime} K x_{1}\right]=p$, the equations (6.5) are consistent and a solution $\beta_{1}$ exists. Also the rank of $\left[M \beta_{1}\right]$ will be $p+1$, for if we assume that there is a column vector " $\alpha$ " having $p$ elements such that $M a=\beta_{1}$, then (6.5) gives $x_{1}=M^{\prime} K M \alpha=0$. Now consider the linear equations

$$
\left[\begin{array}{ll}
M & B_{1}
\end{array}\right]^{\prime} K \beta_{2}=\left[\begin{array}{l}
x_{2} \\
y_{12}
\end{array}\right]
$$

where $y_{i j}$ is the element in the $i$ th row and $j$ th column of $Y$. Again this system is consistent and a solution $\beta_{2}$ exists such that the rank of $\left[M \beta_{1} \beta_{2}\right]$ is $p+2$. Further,

$$
\left[\beta_{1} \beta_{2}\right]^{\prime} K\left[\beta_{1} \beta_{2}\right]=\left[\begin{array}{cc}
0 & y_{12} \\
-y_{12} & 0
\end{array}\right] .
$$

Proceeding in this way, we find that the solutions of

$$
\left[M \beta_{1} \cdots \beta_{i}\right]^{\prime} K \beta_{i+1}=\left[x_{i+1}^{\prime}, y_{1, i+1} \cdots y_{i, i+1}\right]^{\prime}
$$

exist and the rank of $\left[M \beta_{1} \ldots \beta_{i} \beta_{i+1}\right]$ is $p+i+1$ for $i=1,2, \ldots, p-1$. Let $N=\left[\beta_{1} \cdots \beta_{p}\right]$. Then $[M N]$ has rank $2 p$ and $M^{\prime} K N=X$ and $N^{\prime} K N=Y$.

THEOREM 6.6. Let $M_{1} y[0, \infty]=0, M_{2} y[0, \infty]=0$ be two real selfadjoint boundary conditions. Then there exists $a_{v} J_{r}$-symplectic matrix $S$ such that $M_{1}=S M_{2}$.

Proof. Applying Theorem 6.5 to $M_{1}$ and $M_{2}$ with $X=-I$ and $Y=0$, we can find $(n+2 r) \times(\nu+r)$ matrices $N_{1}$ and $N_{2}$ such that $\left[M_{1} N_{1}\right]$ and $\left[M_{2} N_{2}\right]$ are nonsingular and

$$
M_{1}^{\prime}{ }_{v} J_{r} N_{1}=-I, \quad M_{2}^{\prime}{ }_{v} J_{r} N_{2}=-I, \quad N_{1}^{\prime}{ }_{v} J_{r} N_{1}=N_{2}^{\prime}{ }_{v} J_{r} N_{2}=0 .
$$

Since the matrices ${ }_{v} J_{r}$ and $M_{1}$ and $M_{2}$ are real, we can take $N_{1}$ and $N_{2}$ to be real, so that

$$
\left[M_{1} N_{1}\right]^{\prime}{ }_{v} J_{r}\left[M_{1} N_{1}\right]=\left[M_{2} N_{2}\right]^{\prime}{ }_{v} J_{r}\left[M_{2} N_{2}\right]=J_{v+r}
$$

Hence, the matrix $S=\left[M_{1} N_{1}\right]\left[M_{2} N_{2}\right]^{-1}$ is ${ }_{v} J_{r}$-symplectic and $M_{1}=S M_{2}$.

Finally we proceed to prove Lemma 2.3 of [2] in the following section.

7. Extension of a lemma due to Titchmarsh. We assume that the number of square-integrable solutions are $\nu+r$. Consider the real selfadjoint boundary conditions $M y[0, \infty)=0$ where the real matrix $M$ is given by

$$
M=\left[\begin{array}{cc}
\chi_{1} & 0 \\
0 & \chi_{2}
\end{array}\right]
$$


where $\chi_{1}$ is $n \times \nu$ and $\chi_{2}$ is $2 r \times r$. For nonreal $\mu$, we choose a set of $\nu$ linearly independent solutions of $L y+\mu y=0$, denoted by $\phi(x, \mu)$, which satisfy the $\nu$ initial conditions of $M y[0, \infty)=0$. We can choose another set of $\nu$ solutions $\theta(x, \mu)$ such that $[\theta(x, \mu) \phi(x, \mu)]$ form a fundamental set of solutions such that if $W[\theta(x, \mu)]$ $=F(x, \mu)$ and $W[\phi(x, \mu)]=H(x, \mu)$ then $[F H]^{\prime}(x, \mu) B(x)[F H](x, \mu)=J_{v}$.

We shall now find a matrix $Z(\mu)$ such that the set of solutions $\psi(x, \mu)=\theta(x, \mu)$ $+\phi(x, \mu) Z(\mu)$ satisfy the $r$ final conditions, so that if $W[\psi(x, \mu)]=G(x, \mu)$ then

$$
\operatorname{Lt}_{x \rightarrow \infty} G^{*}(x, \mu) B(x) G(x, \mu)=0 .
$$

We have seen in $\S 3$ that any $Z(\mu)$ satisfying (7.1) is of the form

$$
Z(\mu)=(1 / 2 v) P(\mu) \mathscr{U} P^{\prime}(\mu)+\mathscr{C}(\mu)
$$

with the usual notations. If $\mathscr{U}_{1}$ is the first $r$ th ordered principal minor of $\mathscr{U}$ then

$$
Z(\mu)=(1 / 2 v) \mathscr{P}(\mu) \mathscr{U}_{1} \mathscr{P}^{\prime}(\mu)+\mathscr{C}(\mu) .
$$

Only $\mathscr{U}_{1}$ is unknown on the right side of (7.2) and we proceed to find $Z(\mu)$ so that $\psi(x, \mu)$ satisfies the $r$ conditions at infinity.

Since $t(x)$ is a basis of $D^{*} \bmod D_{0}$, there exists a matrix $N(\mu)$,

$$
N(\mu)=\left[\begin{array}{ll}
N_{11}(\mu) & N_{12}(\mu) \\
N_{21}(\mu) & N_{22}(\mu)
\end{array}\right]
$$

where $N_{11}$ is $n \times r, N_{21}$ is $2 r \times r, N_{12}$ is $n \times \nu$ and $N_{22}$ is $2 r \times \nu$, such that

$$
[\phi(x, \mu) \mathscr{P}(\mu) \theta(x, \mu)+\phi(x, \mu) \mathscr{C}(\mu)]=t(x) N(\mu) .
$$

Since $\phi(x, \mu)$ satisfies the conditions at zero,

$$
\left[\chi_{1} 0\right]\left[\begin{array}{cc}
J_{v} & 0 \\
0 & 0_{2 r}
\end{array}\right]\left[\begin{array}{l}
N_{11}(\mu) \\
N_{21}(\mu)
\end{array}\right]=0
$$

THEOREM 7.1. The matrix $\left[\chi_{2}^{\prime} J_{r} N_{21}\right](\mu)$ is nonsingular for nonreal $\mu$.

Proof. We shall show that if $\left[\chi_{2}^{\prime} J_{r} N_{21}\right](\mu)$ is singular for any $\mu$ then $\mu$ is real. We have

$$
\left[\chi_{2}^{\prime} N_{21}(\mu)\right]^{\prime} J_{r}\left[\chi_{2} N_{21}(\mu)\right]=\left[\begin{array}{cc}
0 & \chi_{2}^{\prime} J_{r} N_{21}(\mu) \\
N_{21}^{\prime}(\mu) J_{r} \chi_{2} & N_{21}^{\prime}(\mu) J_{r} N_{21}(\mu)
\end{array}\right]
$$

If $\left[\chi_{2}^{\prime} J_{r} N_{21}\right](\mu)$ is singular, then the matrix on the right will be singular so that the matrix on the left will be singular. Hence, there exist nonzero column vectors $\alpha$ and $\beta$ such that $\chi_{2} \alpha=N_{21}(\mu) \beta, \beta \neq 0$, for, if $\beta=0$, then since $\chi_{2}$ has rank $r, \alpha=0$. Hence, the solution

$$
\alpha(x, \mu)=\phi(x, \mu) \mathscr{P}(\mu) \beta=t(x)\left[\begin{array}{l}
N_{11} \mu \\
N_{21} \mu
\end{array}\right] \beta
$$

is nontrivial and satisfies the conditions at infinity and by (7.3) also satisfies the 
conditions at zero. Hence, $\alpha(x, \mu)$ is an eigenfunction and $\mu$ is an eigenvalue so that $\mu$ will be real. Now

$$
\begin{aligned}
\psi(x, \mu) & =\theta(x, \mu)+\phi(x, \mu) \mathscr{C}(\mu)+(1 / 2 v) \phi(x, \mu) \mathscr{P}(\mu) \mathscr{U}_{1} \mathscr{P}^{\prime}(\mu) \\
& =t(x) N(\mu)\left[\begin{array}{c}
\mathscr{U}_{1} \mathscr{P}^{\prime}(\mu) \\
I_{v}
\end{array}\right] .
\end{aligned}
$$

If these solutions are to satisfy the conditions at infinity,

$$
\left[\begin{array}{ll}
0 & \chi_{2}^{\prime}
\end{array}\right]\left[\begin{array}{cc}
0_{n} & 0 \\
0 & J_{r}
\end{array}\right] N(\mu)\left[\begin{array}{c}
\mathscr{U}_{1} \mathscr{P}^{\prime}(\mu) \\
I_{v}
\end{array}\right]=0
$$

i.e.,

$$
\chi_{2}^{\prime} J_{r}\left[N_{21}(\mu) \mathscr{U}_{1} \mathscr{P}^{\prime}(\mu)+N_{22}(\mu)\right]=0
$$

or

$$
\left[\left(\chi_{2}^{\prime} J_{r} N_{21}\right) \mathscr{U}_{1} \mathscr{P}^{\prime}\right](\mu)=-\left(\chi_{2}^{\prime} J_{r} N_{22}\right)(\mu)
$$

Since $\operatorname{Im} \mu \neq 0$,

$$
\mathscr{U}_{1} \mathscr{P}^{\prime}(\mu)=-\left[\left(\chi_{2}^{\prime} J_{r} N_{21}\right)^{-1}\left(\chi_{2}^{\prime} J_{r} N_{22}\right)\right](\mu) .
$$

The matrix $Z(\mu)$ can now be found by (7.2).

THEOREM 7.2. $\mathrm{Lt}_{x \rightarrow \infty} B\left[\psi(x, \mu), \psi\left(x, \mu^{\prime}\right)\right]=0$.

Proof. Since $\psi(x, \mu)$ and $\psi\left(x, \mu^{\prime}\right)$ satisfy the same set of conditions at infinity, we have $\chi_{2}^{\prime} J_{r}\left[N_{21}(\mu) \mathscr{U}_{1} \mathscr{P}^{\prime}(\mu)+N_{22}(\mu)\right]=0$ and a similar expression for $\mu^{\prime}$ with $\mathscr{U}_{2}$ instead of $\mathscr{U}_{1}$.

From these we have

$$
\left[N_{21}\left(\mu^{\prime}\right) \mathscr{U}_{2} \mathscr{P}^{\prime}\left(\mu^{\prime}\right)+N_{22}\left(\mu^{\prime}\right)\right]^{\prime} J_{r}\left[N_{21}(\mu) \mathscr{U}_{1} \mathscr{P}^{\prime}(\mu)+N_{22}(\mu)\right]=0 .
$$

This is the required result, for, since

$$
\psi(x, \mu)=t(x) \cdot N(\mu)\left[\begin{array}{c}
\mathscr{U}_{1} \mathscr{P}^{\prime}(\mu) \\
I_{v}
\end{array}\right]
$$

and

$$
\psi\left(x, \mu^{\prime}\right)=t(x) \cdot N\left(\mu^{\prime}\right)\left[\begin{array}{c}
\mathscr{U}_{2} \mathscr{P}^{\prime}\left(\mu^{\prime}\right) \\
I_{v}
\end{array}\right]
$$

$\operatorname{Lt}_{x \rightarrow \infty} B\left[\psi(x, \mu), \psi\left(x, \mu^{\prime}\right)\right]$

$$
\begin{aligned}
& =\left[\mathscr{P}\left(\mu^{\prime}\right) \mathscr{U}_{2} I_{v}\right] N^{\prime}\left(\mu^{\prime}\right)\left[\begin{array}{cc}
0_{n} & 0 \\
0 & J_{r}
\end{array}\right] N(\mu)\left[\begin{array}{c}
\mathscr{U}_{1} \mathscr{P}^{\prime}(\mu) \\
I_{\nu}
\end{array}\right] \text { by }(5.14) \\
& =\left[\mathscr{P}\left(\mu^{\prime}\right) \mathscr{U}_{2} I_{v}\right]\left[\begin{array}{l}
N_{21}^{\prime}\left(\mu^{\prime}\right) \\
N_{22}^{\prime}\left(\mu^{\prime}\right)
\end{array}\right] J_{r}\left[N_{21}(\mu) N_{22}(\mu)\right]\left[\begin{array}{c}
\mathscr{U}_{1} \mathscr{P}^{\prime}(\mu) \\
I_{v}
\end{array}\right]=0 \quad \text { by (7.4). }
\end{aligned}
$$




\section{REFERENCES}

1. W. N. Everitt, Singular differential equations. II: Some self-adjoint even order cases, Quart. J. Math. Oxford Ser. (2) 18 (1967), 13-32. MR 35 \#442.

2. E. C. Titchmarsh, Eigenfunction expansions associated with second-order differential equations, Clarendon Press, Oxford, 1946. MR 8, 458.

3. E. A. Coddington and N. Levinson, Theory of ordinary differential equations, McGrawHill, New York, 1955. MR 16, 1022.

4. R. Courant and D. Hilbert, Methods of mathematical physics. Vol. I, Interscience, New York, 1953. MR 16, 426.

5. W. N. Everitt, Some properties of Gram matrices and determinants, Quart. J. Math. Oxford Ser. (2) 9 (1958), 87-98. MR 20 \#3172.

6. - Integrable-square solutions of ordinary differential equations, Quart. J. Math. Oxford Ser. (2) 10 (1959), 145-155. MR 21 \#5766.

7. K. Kodaira, On ordinary differential equations of any even order and the corresponding eigenfunction expansions, Amer. J. Math. 72 (1950), 502-544. MR 12, 103.

8. W. N. Everitt, Fourth order singular differential equations, Math. Ann. 149 (1962/63), 320-340. MR 27 \#2668.

9. _- Singular differential equations. I: The even order case, Math. Ann. 156 (1964), 9-24. MR 29 \#3709.

10. I. M. Glazeman, On the theory of singular differential operators, Uspehi Mat. Nauk 5 (1950), no. 6 (40), 102-135; English transl., Amer. Math. Soc. Transl. (1) 4 (1962), 331-372. MR 13, 254.

UNIVERSITY OF MARYLAND,

College Park, Maryland 20742 